\title{
Diversité Inter Décennale De La Végétation De La Vallée De Goulbi N'Kaba
}

\author{
Mahaman Hamissou Illo Souley \\ Université Dan Dicko Dan Koulodo de Maradi, Niger. Faculté d'Agronomie \\ et des Sciences de l'Environnement, Maradi-Niger. \\ Institut Pratique de Développement Rural de Kollo (IPDR/ Kollo). \\ Département Élevage, IPDR/ Kollo, Kollo-Niger

\section{Karim Saley} \\ Université Dan Dicko Dan Koulodo de Maradi.
}

Faculté des Sciences et Techniques, Département de Biologie, Maradi-Niger

\section{Issa Chaibou}

Université Dan Dicko Dan Koulodo de Maradi, Niger.

Faculté d'Agronomie et des Sciences de l'Environnement,

Département Sciences et Techniques d'Élevage, Maradi-Niger

\section{Boubacar M. Moussa}

Université de Diffa. Faculté des Sciences Agronomiques.

Phd Éco-pédologie, Diffa Niger

\section{Ali Mahamane}

Université de Diffa. Professeur Titulaire, Enseignant chercheur, Recteur de l'Université de Diffa

\section{Mahamane Saadou}

Université de Niamey. Professeur Titulaire, Enseignant chercheur

\section{Doi: 10.19044/esj.2018.v14n9p161 URL:http://dx.doi.org/10.19044/esj.2018.v14n9p161}

\section{Abstract}

The present study is conducted in the Sylvo-Pastoral Zone (ZSP) of the Goulbi N'Kaba Valley and the agricultural zone (fields on adjacent terraces), in the southern center of Niger. The objective of the study is to evaluate the inter-decennial plant biodiversity (2004-2014). Thus, the phytosociological surveys (Daget and Poissonet, 1971) were carried out in plots of $2500 \mathrm{~m}^{2}$, along the $6.5 \mathrm{~km}$ transects following stratified sampling. An ascending hierarchical classification, followed by an ordination by Nonmetric Multi-dimensional Scaling (NMS) allowed to identify the plant groups. The diversity of each group was evaluated. The results show that the families of Poaceae and Fabaceae are the most abundant in 2004 (15.58\% and 11.69\%) and in 2014 (18.75\% and 7.29\%). However, compared to the genera, 
Indigofera (10.53\%), Cassia (6.58\%), Acacia (5.26\%), Aristida (5.26\%) are the most abundant in 2004, while Aristida (4 , 17\%), Cassia (4.17\%), Spermacoce (3.13\%), in 2014. Three groups (mixed, fields, ZSP) have been identified. The 2004 and 2014 index, of Margalef (18,38 and 11,28), Menhinick (2,55 and 1,52), Shannon-Weaver (4,77 and 4,14), the fairness of Pielou (0, 66 and 0.63), the Simpson index (0.89 and 0.91) and Hill (0.95 and 0.93 ) are high in all the groups, mixed, fields and ZSP (respectively for G1, G2 and G3). But the beta diversity (Whittaker (24), Jaccard (0.52), Sorensen (0.69) index) for each of these groupings remains low. There was no significant change in inter-decennial diversity at the Guidan Tawayé and Korin Habdjia sites from 2004 to 2014.

Keywords: Goulbi N'Kaba, ZSP, Fields, Diversity, Inter-decennial

\section{Résumé}

La présente étude est conduite dans la zone sylvo-pastorale (ZSP) et la zone agricole (champs sur les terrasses adjacentes) de de la vallée de Goulbi N'Kaba, au centre sud du Niger. L'objectif de l'étude est d'évaluer la biodiversité végétale interdécennale (2004-2014). Ainsi, les relevés phytosociologiques (Daget et Poissonet, 1971) ont été effectués dans des placettes de $2500 \mathrm{~m}^{2}$, le long des transects de $6,5 \mathrm{~km}$ suivant l'échantillonnage stratifié. Une classification hiérarchique ascendante, suivie d'une ordination par Nonmetric Multidimensional Scaling (NMS) ont permis d'identifier les groupements végétaux. La diversité de chaque groupement a été évaluée. Les résultats montrent que les familles des Poaceae et des Fabaceae sont les plus abondantes en $2004(15,58 \%$ et $11,69 \%)$ et en $2014(18,75 \%$ et 7,29\%). Cependant, par rapport aux genres, Indigofera (10,53\%), Cassia (6,58\%), Acacia $(5,26 \%)$, Aristida (5,26\%) sont les plus abondants en 2004, tandis que Aristida (4,17\%), Cassia (4,17\%), Spermacoce $(3,13 \%)$, en 2014. Trois groupements (mixte, champs, ZSP) ont été identifiés. Les indices 2004 et 2014 , de Margalef $(18,38$ et 11,28), Menhinick (2,55 et 1,52), ShannonWeaver $(4,77$ et 4,14$)$, l'équitabilité de Pielou $(0,66$ et 0,63$)$, l'indice de Simpson $(0,89$ et 0,91$)$ et Hill $(0,95$ et 0,93$)$ sont élevés dans tous les groupements, mixte, champs et ZSP (respectivement pour G1, G2 et G3). Mais la diversité beta (indices de Whittaker $(24)$, Jaccard $(0,52)$, Sorensen $(0,69)$ ) pour chacun de ces groupements reste faible. Il n'y a pas eu un changement notable de diversité interdecénnale sur les sites de Guidan-Tawayé et KorinHabdjia de 2004 à 2014.

Mots clés : Goulbi N'Kaba, ZSP, Champs, Diversité, Interdécennale 


\section{Introduction}

Les régions sahéliennes sont caractérisées au cours de ces dernières décennies par une forte variabilité annuelle et spatiale de la pluviosité et une tendance à l'aridité progressive du climat (Jouve, 1991), ce qui n'est pas sans conséquence sur la biodiversité. Le Niger, pays sahélien situé dans l'Afrique Occidentale, recèle d'importantes potentialités en matière de diversité biologique et de la diversité inter et intra spécifique de la flore et de la faune (Alzouma, 1998, Saadou, 1998). La biodiversité végétale du Niger comprend 2274 espèces, dont le groupe inférieur avec 133 espèces $(5,85 \%), 547$ espèces d'Algues (24,05\%), 24 espèces de Bryophytes et Ptéridophytes (1,06\%), 1570 espèces $(69,04 \%)$ d'Angiospermes repartis en 444 classes de Monocotylédones et 1016 classes de Dicotylédones (Ahmed et Rouscoua , 2001; Saadou, 1998 ; Le Houérou, 1997 ). Ce dernier groupe est soumis à une exploitation voire une surexploitation (Sonké, 1998 ; Chapman, 1995) pour l'alimentation humaine (plus de 310 espèces) (Saadou, 1998), l'alimentation animale (235 espèces), la pharmacopée traditionnelle (270 espèces), 1'artisanat (127 espèces), la culture etc. (Saadou, 1998 ; Saadou et Garba, 1997).

À ceux-là s'ajoute la poussée démographique, l'augmentation du cheptel, l'extension incontrôlée des emblavures, les déboisements massifs et les effets néfastes des sécheresses aggravés par des déficits pluviométriques moyens sont autant des facteurs qui contribuent globalement à la dégradation accélérée des ressources naturelles (Chamard et Courel, 1999 ; Sar et al., 2013). Il en résulte une perturbation ou la perte des habitats naturels, la rareté voire l'extinction des espèces locales de grande valeur socioéconomique et écologique et leur remplacement par des espèces invasives, et l'accentuation des effets néfastes du changement climatique. En effet, la vallée de Goulbi N'Kaba n'échappe pas à ces phénomènes, la perturbation des écosystèmes se traduit par une prolifération de Sida cordifolia au niveau des espaces émergés et Acanthospermum hispidum au niveau des terres immergées induisant une diminution de l'intérêt pastoral des parcours de la zone. L'étude de la dynamique de la végétation permet en outre de rendre compte de l'évolution du milieu, mais aussi parfois d'actualiser les typologies existantes (Soumana et al., 20016).

La végétation de la vallée de Goubli N'Kaba a fait l'objet de nombreux travaux sur la pré-identification des douméraies (Saley et Seydou, 1989), leur caractérisation (Ichaou, 2005). Peltier et al (2008) ont estimé la quantité et la qualité des feuilles, stipes et fruits de doum et celle du volume exploitable des espèces forestières autres que le doum. Saadou en 2004 a fait l'état des lieux de la diversité végétale le long des transects (des massifs Goulbi N'Kaba) et a mis en place un dispositif de suivi. Suivre l'évolution de cette diversité a longtemps été négligée. L'objectif de cette étude vise à connaitre l'état de la 
biodiversité végétale, caractériser les différents groupements végétaux et analyser leurs diversités (alpha et beta) interdécennales (2004-2014).

\section{Matériel et méthodes \\ Site d'étude}

Cette étude a été conduite le long de la vallée de Goulbi N'Kaba sur deux (2) sites situés à $10 \mathrm{~km}$ à l'est et à $30 \mathrm{~km}$ à l'ouest du chef-lieu de département de Mayahi (région de Maradi) dans les terroirs de Guidan Tawayé $\left(13^{\circ} 89^{\prime} \mathrm{N}\right.$ et $\left.7^{\circ} 60^{\prime} \mathrm{E}\right)$ et Koren Habdjia $\left(13^{\circ} 92^{\prime} \mathrm{N}\right.$ et $\left.7^{\circ} 48^{\prime} \mathrm{E}\right)$ (Figure 1$)$. Les altitudes moyennes des sites sont respectivement de $379,33 \pm 6,29 \mathrm{~m}$ et $369,89 \pm 6,13 \mathrm{~m}$. Le choix des sites du massif forestier de Goulbi N'Kaba (Saadou, 2004) a été fait sur la base d'actualisation de l'état des lieux de la diversité végétale le long des transects, matérialisé depuis 2003 (phase pilote) par le PAFN (Projet d'Aménagement des Forêts Naturelles), dans le but d'assurer la gestion durable des ressources forestières.

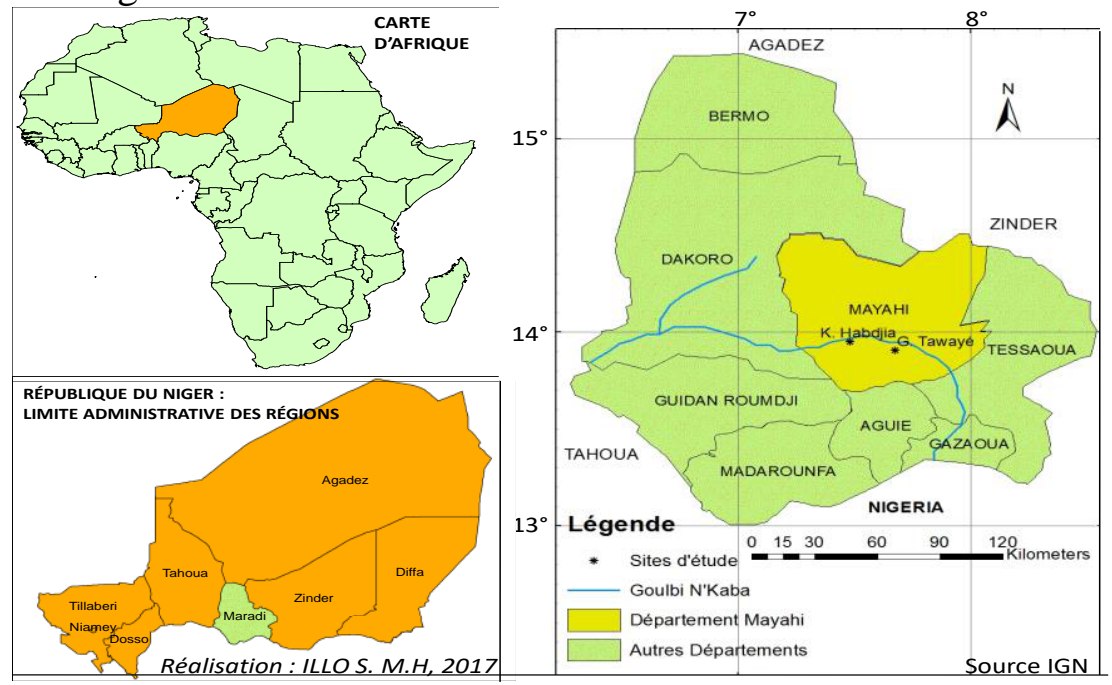

Figure 1 : Carte de localisation de sites d'étude (Guidan Tawayé- Korin Habdjia)

Le climat est tropical aride de type sahélien (Killiam et Feau, 1976) limitée par les isohyètes 400 et $600 \mathrm{~mm}$, caractérisé par une longue saison sèche ( 8 mois) et une courte saison pluvieuse (4mois). Les mois d'août et de juillet sont les plus pluvieux avec $132,88 \pm 67,89 \mathrm{~mm}$ et $167,27 \pm 73,37 \mathrm{~mm}$ (Figure 2). Les températures minimale et maximale de 1970 à 2015 (45 dernières années) sont respectivement en moyenne de $20,78 \pm 4,41^{\circ} \mathrm{C}$ et $34,91 \pm 3,29^{\circ} \mathrm{C}$ (Figure 2). 




Figure 2 : Climogramme (1970-2015) de la région de Maradi (station synoptique de référence Maradi).

La population de Guidan Tawayé est de 3866 et celle de Koren Habdjia est de 2816 habitants (INS, 2014). Elle pratique l'élevage sur le lit mineur de la vallée du Goulbi N'Kaba qui est la zone sylvo-pastorale (ZSP), alors que les berges et les terrasses adjacentes sont destinées à l'agriculture. La végétation est constituée de steppe arbustive et arborée à trois strates. Une strate herbacée composée surtout de: Eragrostis tremula Steud., Cassia mimosoides L., Schoenefeldia gracilis Kunth., Acanthospermum hispidum DC.,etc.; Une strate arbustive constituée de Calotropis procera (Ait.) R. Br., Ziziphus mauritiana Lam., Balanites aegyptiaca (L.) Del., Bauhinia rufescens Lam., Leptadenia pyrotechnica (Forsk.) Decne. etc.; et une strate arborescente dont les espèces dominantes sont Faidherbia albida (Del.) A. Chev., Acacia tortilis (Forsk.) Hayne subsp. raddiana (Savi.) Brenan., Hyphaene thebaica (L.) Mart., Acacia nilotica (L.) Willd. ex. Del. var adansonii, etc. (Saadou et al., 2004).

\section{Collecte et analyse des données}

Le dispositif expérimental a consisté en des transects de $6 \mathrm{~km}$, le long desquels ont été régulièrement placés des placettes de $2500 \mathrm{~m}^{2}$ de relevés par la méthode de points quadrats alignés de Daget et Poissonet, 1971 pour les herbacées (Daget et Poissonet, 1971 in Daget et Poissonet, 1991 ; Gounot, 1969) et le comptage direct pour les ligneux. 


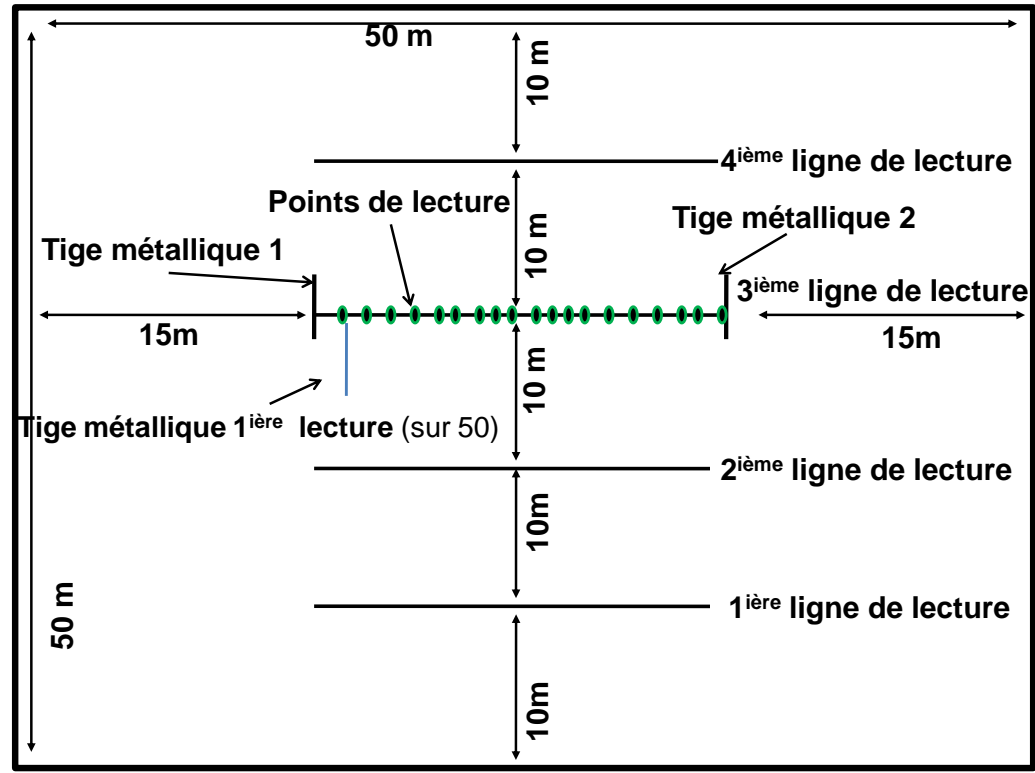

Figure 3 : Schéma du dispositif de la méthode de points quadrats alignés (Daget et Poissonet, 1971) modifiée (Saadou, 2004).

\section{Analyses statistiques}

L'ordination par NMS, qui est une méthode d'évaluation de la dimensionnalité d'un ensemble de données a d'abord été effectuée pour une meilleure approximation des distances observées entre les relevés (Clarke et Ainsworth, 1993). L'appréciation du Stress selon la règle de Clarke, 1993 est le critère qui permet de savoir si la représentation est fidèle ou non. Une valeur de Stress supérieure à 50\% décrit une représentation probablement aléatoire ; entre 25 et $50 \%$, de qualité médiocre ; entre 10 et $25 \%$, satisfaisante ; en dessous de 10\%, excellente (Grall \& Hily, 2003). Le "Stress de Kruskal" (Grall $\&$ Hily, 2003) est représenté par une courbe de Scree. Ensuite la classification hiérarchique en utilisant la distance euclidienne et la méthode de Ward pour distinguer les différents groupements. Enfin Indicator Species Analysis (ISA) (Dufrêne \& Legendre, 1997) a été utilisé pour identifier les espèces caractéristiques des groupements avec le test de Monte Carlo (Mc Cune et al., 2002). Deux espèces (ligneuse et herbacée) ayant la plus grande VI et la plus faible p-value. Le logiciel PC-ORD 5 a été utilisé pour l'ordination et le test ISA. Enfin, le test de comparaison de deux (2) proportions avec le logiciel R ( $\mathrm{R}$ development Core T., 2010), ont été effectués sur les paramètres des groupements (familles, genres, indices) pour apprécier l'évolution de la biodiversité. 


\section{Calculs des indices de diversités biologiques}

La richesse floristique $(\mathrm{S})$, qui représente le nombre total d'espèces a été déterminée (Magurran, 2004). Pour tenter de corriger la richesse spécifique au sein des échantillons ayant un nombre différents d'espèces identiques, il a été proposé des indices de diversité de Margalef et de Menhinick (Soudant et Belin, 2011 ; Magurran, 2004).

Margalef : $\boldsymbol{D}_{\boldsymbol{M g}}=\frac{(\boldsymbol{S}-\mathbf{1})}{\ln (\boldsymbol{N})}$

Menhinick : $\boldsymbol{D}_{\boldsymbol{M n}}=\frac{\boldsymbol{S}}{\sqrt{\boldsymbol{N}}}$

$\mathrm{S}$ : Nombre total d'espèces

$\mathrm{N}$ : Abondance totale des espèces.

Ces indices jouent un rôle important dans la recherche de la diversité biologique. Les diversités Alpha et Beta de différents groupes ont été déterminées.

\section{Diversité Alpha}

Legendre et Legendre (1998) propose l'utilisation de l'anthropie H, comme indice de diversité. L'indice de diversité Alpha de Shannon-Weaver 1949 a été calculé par la formule suivante (Magurran, 2004) :

$H=-\sum_{i=1}^{n} P_{i} . \log _{2} P_{i}$

Les valeurs de $\mathrm{H}$ sont exprimées en bits et varient de 1 à 5 . Les faibles valeurs de $\mathrm{H}$, traduisent les conditions défavorables du milieu pour l'installation des espèces.

L'indice d'équitabilité ou de régularité de Pielou 1969 évalue le poids de chaque espèce dans l'occupation de l'espace (Magurran, 2004 ; D'Avignon et al., 2000). Il est d'autant plus élevé qu'un grand nombre d'espèces participe au recouvrement (Grall et Coïc, 2005).

$E($ bit $)=\frac{H}{H_{\max }}$, et $\operatorname{Hmax}=H^{\prime}=\log _{2} S$

La valeur de l'indice varie de 0 (une espèce dominant très largement toutes les autres) à $\log \mathrm{S}$ (lorsque toutes les espèces ont même abondance), $\mathrm{S}$ étant le nombre d'espèces.

L'indice de Simpson ou l'indice de diversité de Simpson mesure la probabilité que deux individus sélectionnés au hasard appartiennent à la même espèce (Grall et Hily, 2003).

$\widetilde{D}=1-D=1-\sum \frac{N_{i}\left(N_{i}-1\right)}{N(N-1)}$, avec $D=\sum \frac{N_{i}\left(N_{i}-1\right)}{N(N-1)}$

$\mathrm{n}_{\mathrm{i}}=$ nombre d'individus dans l'espèce $\mathrm{i}$; et $\mathrm{N}=$ nombre total d'individus. 
L'indice de diversité de Simpson représenté par 1-D, le maximum de diversité étant représenté par la valeur 1, et le minimum de diversité par la valeur 0 . Il donne plus de poids aux espèces abondantes qu'aux espèces rares.

L'indice de diversité de Hill : Hill 1973 (Magurran, 2004 ; Hill, 1997) a proposé une série d'indices dérivés des indices précédents, dont les indices notés $\mathrm{N}_{1}$ et $\mathrm{N}_{2}$ permettant d'appréhender les questions de mesure de la diversité dans la plupart des cas.

Hill $=\frac{N_{2}}{N_{1}}=\frac{(1 / \lambda)}{e^{H^{\prime}}}$ avec $N_{1}=e^{H^{\prime}}$ et $N_{2}=\frac{1}{\lambda} ; \lambda=\sum_{i=1}^{S} p_{i}^{2} \quad$ (Grall et Coïc, 2005) ou

$p_{i}=$ proportion des individus dans l'espèce $\mathrm{i}$;

$\frac{1}{\lambda}=\frac{1}{\widetilde{D}}:$ mesure le nombre effectif d'individus très abondants (Hill, 1997);

$\mathrm{e}^{\mathrm{H}^{\prime}}$ : mesure en revanche le nombre effectif d'individus abondants mais surtout des espèces rares.

Pour faciliter l'interprétation, l'indice 1-Hill est utilisé, où la diversité maximale sera représentée par la valeur 1, et la diversité minimale par la valeur 0 .

Toutefois, il peut être utile d'utiliser les trois indices conjointement afin d'en extraire un maximum d'informations et de mieux comprendre la structure des communautés (Grall \& Coïc, 2005).

\section{Diversité Béta}

L'indice de Whittaker est un indice simple, très utilisé, il donne une très bonne mesure de la diversité $\beta$, en indiquant la dissimilitude entre plusieurs habitats en termes d'espèce (Soudant et Belin, 2011 ; Magurran, 2004). Il est défini comme suit :

$\beta_{w}=\frac{S}{\bar{\alpha}}$ (sans unité varie de 0 à $\mathrm{S}$ ) $\mathrm{S}$ : est le nombre total d'espèces dans l'espace et dans le temps et $\bar{\alpha}$ la moyenne du nombre d'espèces (Soudant et Belin, 2011).

L'indice de Jaccard, teste la similarité entre deux habitats.

$J=\frac{a}{a+b+c} ; \mathbf{a}:$ représente le nombre d'espèces communes entre les deux groupements ;

b : représente le nombre d'espèces uniques au groupement 1 ;

c : représente le nombre d'espèces uniques au groupement 2 .

Si l'indice $J$ augmente, un nombre important d'espèces se rencontre dans les deux habitats évoquant ainsi que la biodiversité inter habitat est faible.

La diversité béta est appréciée en utilisant le coefficient de similitude de Sørensen (1948). Il permet d'apprécier l'existence des communautés. 
$I_{\text {Sørensen }}=\frac{2 c}{a+b+2 c} \mathbf{a}$ : les espèces uniques au groupement $\mathrm{A} ; \mathbf{b}$ : espèces uniques au groupement $\mathrm{B}$ et $\mathbf{c}$ les espèces communes aux deux groupements. (Magurran, 2004 ; Mahamane, 2005).

\section{Résultats}

\section{Familles, Genres, espèces}

Les 24 relevés effectués en 2004 comptent 40 familles, 148 espèces dont 16 espèces ligneuses et 132 herbacées au sein desquelles il faut déduire 4 espèces cultivées Pennisetum glaucum (L.) R Br., Vigna unguiculata (L.) Walp. Subsp. unguiculata, Sorghum bicolor (L.) Moench. et Hibiscus sabdariffa (L.). Quant aux relevés réalisés dans les mêmes parcelles en 2014, il a été dénombré 30 familles regroupant 94 espèces dont 12 espèces ligneuses et 84 herbacées, auxquelles il faut déduire cinq (5) espèces cultivées : Pennisetum glaucum (L.) R Br., Vigna unguiculata (L.) Walp. Subsp. unguiculata, Sorghum bicolor (L.) Moench., Sesamum indicum L. et Hibiscus sabdariffa L. Les familles des Poaceae (24 espèces ou 15,58\%) et Fabaceae (18 espèces, 11,69\%) sont les plus nombreuses en 2004, les autres n'atteignent guère 8 espèces $(6 \%)$. Dix ans après, les familles, les plus nombreuses sont celles des Poaceae (avec 18 espèces 18,75\%), Fabaceae (7 espèces, 7,29\%), Caesalpiniaceae (7 espèces, 7,29\%), Rubiaceae (6 espèces, 6,25\%). En 2004 ces mêmes espèces comptent 105 genres dont les plus nombreux sont les Indigofera, Cassia, Acacia, Aristida et Corchorus avec respectivement 8 $(10,53 \%), 5(6,58 \%), 4(5,26 \%), 4(5,26 \%), 4(5,26 \%)$ espèces. Quant aux relevés de 2014, il a été dénombré 74 genres dont 4 Aristida, 4 Cassia, 3 Spermacoce avec respectivement 4,17\%;4,17\%;3,13\% (Figure 6).

\section{NMS et groupements}

L'analyse Multidimensionnelle Non Métrique (MNS) a fait ressortir trois (3) groupements.

Le stress final de $11,028 \%$ pour les relevés de 2004 et 12,424\% pour ceux de 2014, proche de 10 est une bonne ordination sans risque réel de tirer de fausses inférences. Le Scree parcelle qui est le tracé de la contrainte finale par rapport au nombre de dimensions en illustre (Figure 4 et 5 : Scree Plot). Les 3 premiers axes de relevés de 2004 contiennent $87,10 \%$ et ceux de 2014 en contiennent 85,68\% des informations, selon la courbe de Scree. La carte factorielle sera représentée sur les deux premiers axes (Axes 1 et 2). 

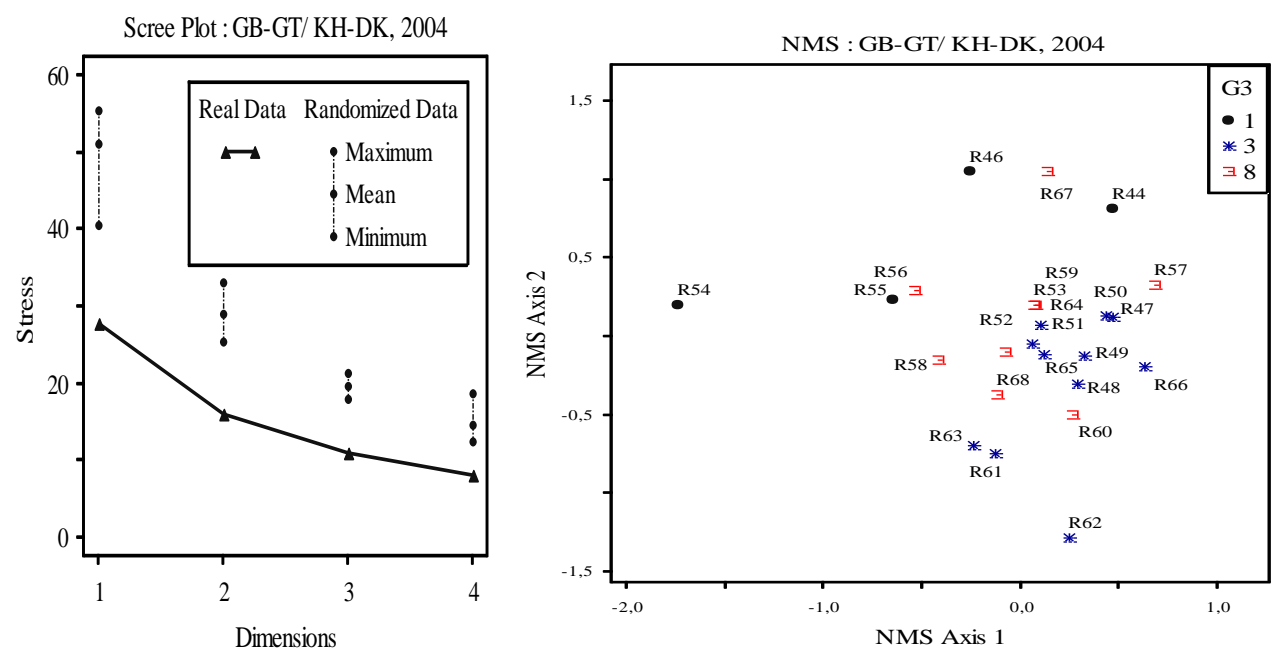

Figure 4 : Représentation de la courbe de Scree et de la carte factorielle NMS dans le plan 1 et 2, de Guidan-Bawa, Guidan-Tawayé et Korin-Habdjia, Dan-Kibia de 2004.
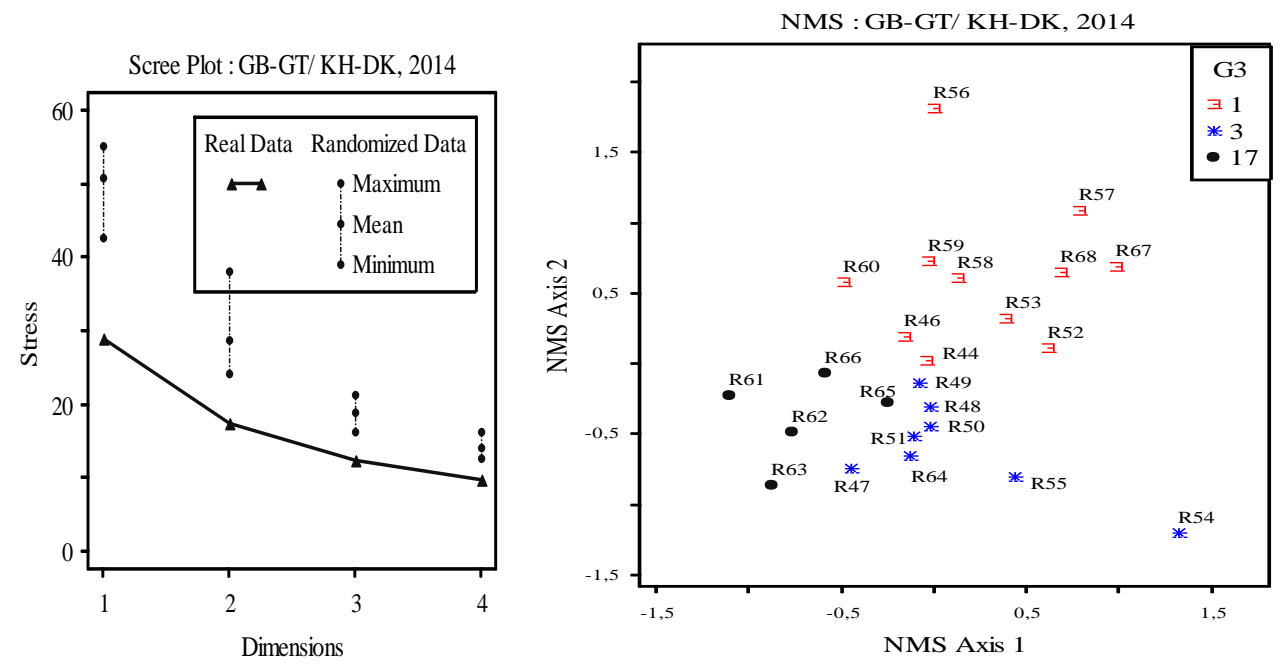

Figure 5 : Représentation de la courbe de Scree, de la carte factorielle NMS dans le plan 1 et 2, de Guidan-Bawa, Guidan-Tawayé et Korin-Habdjia, Dan-Kibia de 2014.

\section{Corrélation (relevés/ groupes)}

En 2004, il apparait ainsi, que les groupes se distinguent par rapport aux axes : l'ensemble de relevés de G1 se situe du côté positif de l'axe NMS2. Le relevé R54 est distant des autres parce qu'il a un recouvrement élevé (189) et il possède le plus grand nombre d'espèces de tous les groupements (60espèces) (Figure 4 : NMS). L'ensemble des relevés de G2 se trouvent du côté positif de l'axe NMS1. Les relevés de G3 se situent du côté positif de l'axe NMS1, sauf le relevé R56 qui leur est opposé (Figure 4 op cit.). Ceci est 
dû au fait que c'est le seul relevé, qu'il possède plus d'espèces (40) que les autres relevés du même groupe.

En 2014, tous les relevés de G1 se trouvent du côté négatif de l'axe NMS21 et NMS2 (Figure 5 : NMS). Ceux de G2 se trouvent du côté positif de l'axe NMS1. Le révélé R56 est distant du groupe parce qu'il a un nombre d'espèces plus élevé (32espèces). L'ensemble de relevés de G3 se situent du côté négatif de l'axe NMS2 (Figure 5 op cit.). Il faut noter que R54 est isolé du groupe G3. Cela est dû au fait qu'il possède plus grand nombre d'espèces (35espèces).

Les relevés individualisés étaient dus au nombre d'espèces rares plus élevés qu'ils en possèdent par rapport aux autres du même groupe.

Distance (relevés/ groupes): En 2004, le relevé R54 bien qu'appartenant à G1, n'est pas proche des autres du même groupe, dans l'espace de représentation contenant l'axe NMS1 et NMS2. Cela s'explique par la proportion d'espèces rares élevée $(12,5 \%: 5$ espèces) qu'il en possède. Quant aux relevés R61, R62 de G3 sont isolés par rapport aux autres du même groupe dans l'espace de représentation contenant l'axe NMS1 (Figure 4 op cit.). Ceci est dû au nombre d'espèces rares ( 9 espèces soit $22,5 \% ; 5$ espèces soit $12,5 \%$ ) élevé qu'il en possède (les autres espèces rares ne dépassant guère 2 espèces soit $5 \%$ ).

Il en est de même en 2014, les relevés R54 et R56 bien qu'appartenant à $\mathrm{G} 3$ et $\mathrm{G} 2$, ne sont pas proche des autres relevés du même groupe dans l'espace de représentation contenant les axes NMS1 et NMS2Cela s'explique par le nombre élevé, respectifs de 35 et 32 espèces qu'ils en possèdent par rapport au nombre moyen de $20 \pm 9$ espèces et $22 \pm 5$ espèces. La répartition des différents groupements est liée surtout à la pratique. C'est ainsi que le groupement $\mathrm{G} 2$ est constitués des relevés des jachères (champs), G3 ceux des ZSP et G1 étant constitué des relevés mixtes (champs-ZSP).

\section{Caractéristiques des groupements (Cluster et analyse ISA)}

La Classification Hiérarchique Ascendante (CHA) ont permis de distinguer 3 groupements au seuil de troncature de $31,25 \%$ pour les relevés de 2004 et $25 \%$ pour ceux de 2014, et à une distance Euclidienne respective de $3,76.10^{2}$ et $2,5.10^{2}$. 
Cluster : GB-GT/ KH-DK, 2004

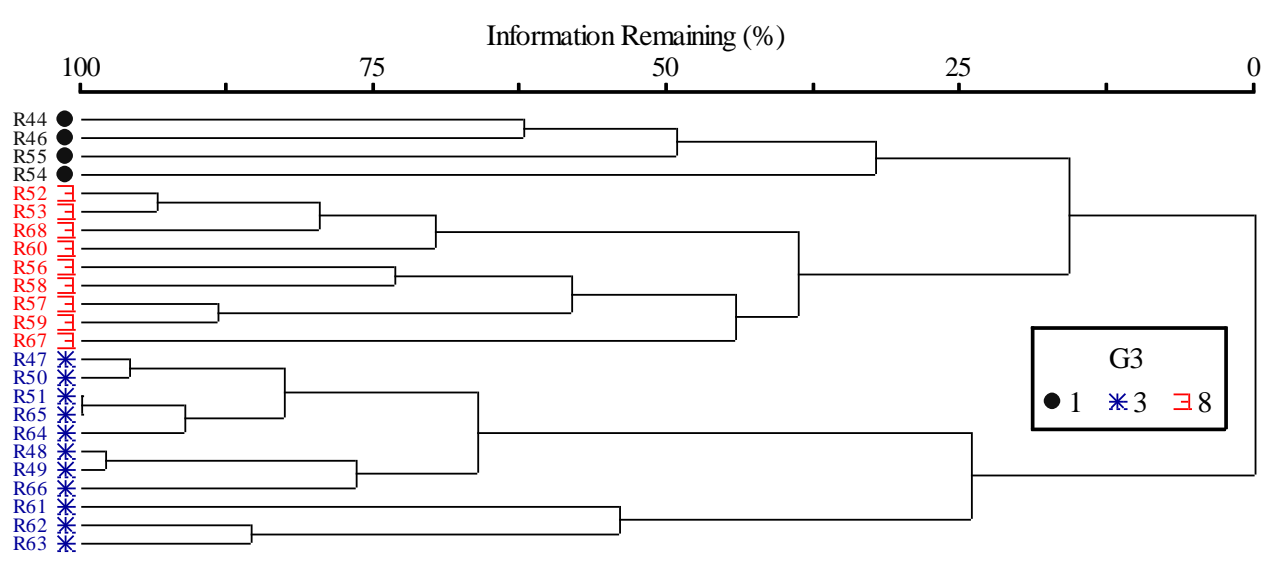

Cluster : GB-GT/ KH-DK, 2014



Figure 6 : Dendrogramme de Guidan-Bawa Guidan-Tawayé et Korin-Habjia Dan Kibia de 2004 et celui de 2014.

Les groupements G1 à Calotropis procera et Jacquemontia tamnifolia (Cp_Jt), G2 à Acacia nilotica et Peristrophe bicalyculata (Ann_Pb), G3 à Leptadenia pyrotechnica et Schoenefeldia gracilis (Lp_Sg) caractérise la répartition des relevés de 2004. Alors que ceux de 2014 sont constitués de groupements G1 à Acacia tortilis et Aristida mutabilis (At_Am), G2 Calotropis procera et Eragrostis tremula (Cp_Et), G3 Balanites aegyptiaca et Glinus oppositifolius (Ba_Go).

Les valeurs indicatrices des différents groupements avec leur p-value issue du test de Monte Carlo sont récapitulées dans le tableau ci-dessous. 
Tableau 1 : Valeurs Indicatrices des groupements de GB-GT et KH-DK de 2004

\begin{tabular}{l|ccccc}
\hline \multicolumn{1}{c|}{ Species 2004 } & Groups & IV & Mean & S. Dev & P* \\
\hline Calotropis procera (Ait.) R. Br. & 1 & 82,7 & 53,1 & 12,56 & 0,023 \\
Jacquemontia tamnifolia (L.) Griseb. & 1 & 92,1 & 42,3 & 12,92 & 0,001 \\
Acacia nilotica (L.) Willd. ex Del. subsp. N & 2 & 38,7 & 22,7 & 9,04 & 0,0801 \\
Peristrophe bicalyculata (Retz.) Nees & 2 & 69,3 & 30 & 12,28 & 0,01 \\
Leptadenia pyrotechnica (Forssk.) Decne. & 3 & 45,3 & 28,1 & 8,74 & 0,06 \\
Schoenefeldia gracilis Kunth. & 3 & 78 & 43,1 & 13,44 & 0,014 \\
\hline
\end{tabular}

Tableau 2 : Valeurs Indicatrices des groupements de GB-GT et KH-DK de 2014

\begin{tabular}{l|ccccc}
\hline \multicolumn{1}{c|}{ Species 2014 } & Groups & IV & Mean & S. Dev & P* \\
\hline Acacia tortilis (forsk.) Hayne & 1 & 42,3 & 34,3 & 6,07 & 0,14 \\
Aristida mutabilis Trin. & 1 & 87,5 & 22,7 & 9,13 & 0,001 \\
Calotropis procera (Ait.) R. Br. & 2 & 43,2 & 22,9 & 8,97 & 0,04 \\
Eragrostis tremula Steud. & 2 & 54,9 & 31,3 & 6,99 & 0,004 \\
Balanites aegyptiaca (L.) Del. & 3 & 21,2 & 22,2 & 8,74 & 0,51 \\
Glinus oppositifolius (L.) A. DC. & 3 & 85,7 & 24,6 & 9,25 & 0,001 \\
\hline
\end{tabular}

En 2004, les formations mixtes steppiques à Calotropis procera (Ait.) R. Br. et Jacquemontia tamnifolia (L.) Griseb. caractérisent du groupe G1. Ensuite les relevés R52, R53, R56, R57, R58, R59, R60, R67, R68 issus du groupe $\mathrm{G} 2$ sont caractérisés par les formations steppiques des champs à Acacia nilotica (L.) Willd. ex Del. subsp. nilotica et Peristrophe bicalyculata (Retz.) Nees. En fin, les formations steppiques des zones sylvopastorales (ZSP) à Leptadenia pyrotechnica (Forssk.) Decne.et Schoenefeldia gracilis Kunth. caractérisent les relevés R47, R48, R49, R50, R51, R61, R62, R63, R64, R65, R66 (Figure 6). Les VI sont supérieures à 69\% pour les espèces herbacées et supérieures à $38 \%$ pour les ligneux (Tableau 1 ).

En 2014, les relevés R61, R62, R63, R65, R66 (2014) du groupe G1 sont caractérisés par les formations steppiques des ZSP à Acacia tortilis (Forsk.) Hayne subsp. raddiana (Savi.) Brenan. et Aristida mutabilis Trin. Les formations des champs steppiques à Calotropis procera (Ait.) R. Br.et Eragrostis tremula Steud. caractérisent les relevés du groupe G2 : R44, R46, R52, R53, R56, R57, R58, R59, R60, R67, R68. Enfin les relevés du groupe G3 : R47, R48, R49, R50, R51, R55, R60, R64 sont caractérisés par les formations steppiques des ZSP à Balanites aegyptiaca (L.) Del. et Glinus oppositifolius (L.) A. DC. (Figure 6 op cit.). Les VI sont supérieures à 54\% pour les espèces herbacées et supérieures à $21 \%$ pour les ligneux (Tableau 2). 


\section{Diversité végétale des sites de Guidan Tawayé et Korin Habdjia de la vallée de Goulbi Kaba 2004 et 2014}

Le groupe G1 (2004) est proche de G1 (2014) qui sont constitués des relevés des champs et des ZSP. Les groupes G2 (2004-2014) sont constitués de relevés des champs, enfin ceux de G3 (2004- 2014) sont constitués uniquement des relevés de la ZSP.

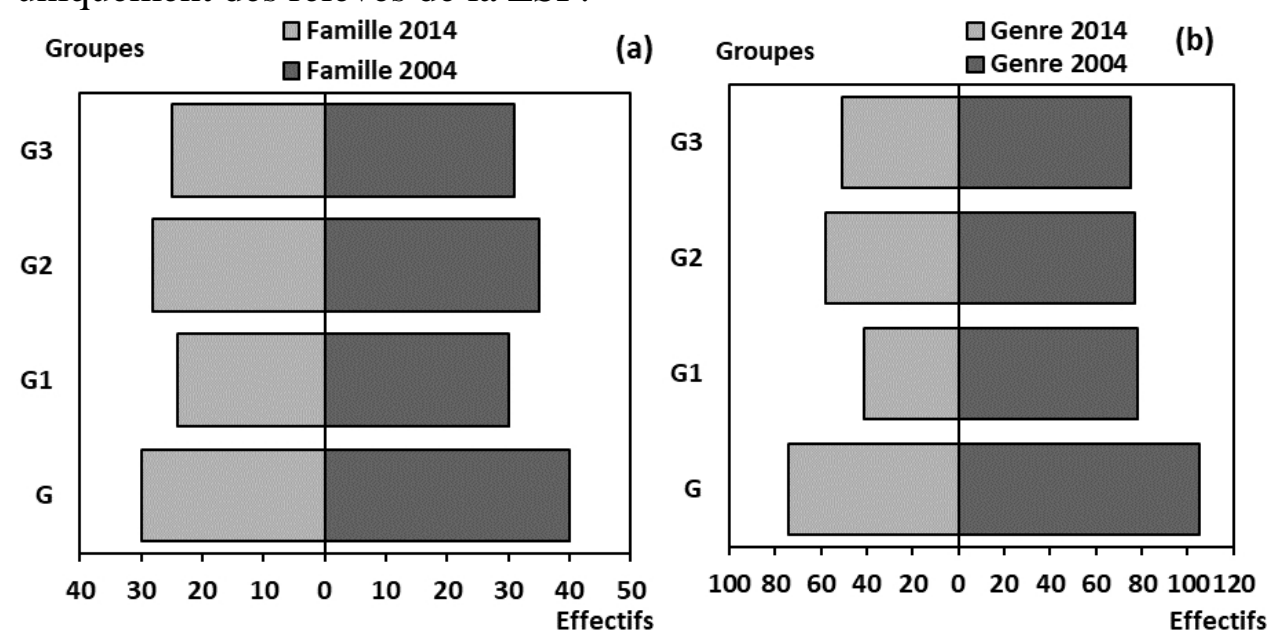

Figure 7 : Le nombre de familles (a) et de genre (b) des sites de 2004 et 2014.

Le nombre de familles de 2004 (40) est supérieur à celui de 2014 (30) (Figure $7:$ : a)). Il en est de même pour les genres de (105 et 74) respectivement pour 2004 et 2014 (Figure 7 : (b)). Il n'y a pas de différence significative dans les proportions de familles inter décennale (2004-2014) des groupes G1, G2 et $\mathrm{G} 3$ car leur $\mathrm{p}$-value respectives 0,$8372 ; 0,6873 ; 0,7627$ sont supérieures à 0,05 (Figure 7 : (a)). Il y a une similarité dans la répartition de genres de groupes G2 et G3 (de 2004 et 2014), avec leurs p-value respectives de 0,5514 et 0,8446 . Mais il y a dissimilarité dans la distribution de G1 (2004- 2014) avec $\mathrm{p}$-value de $0,01335<0,05=\mathrm{p}$.

\section{Diversité Alpha}

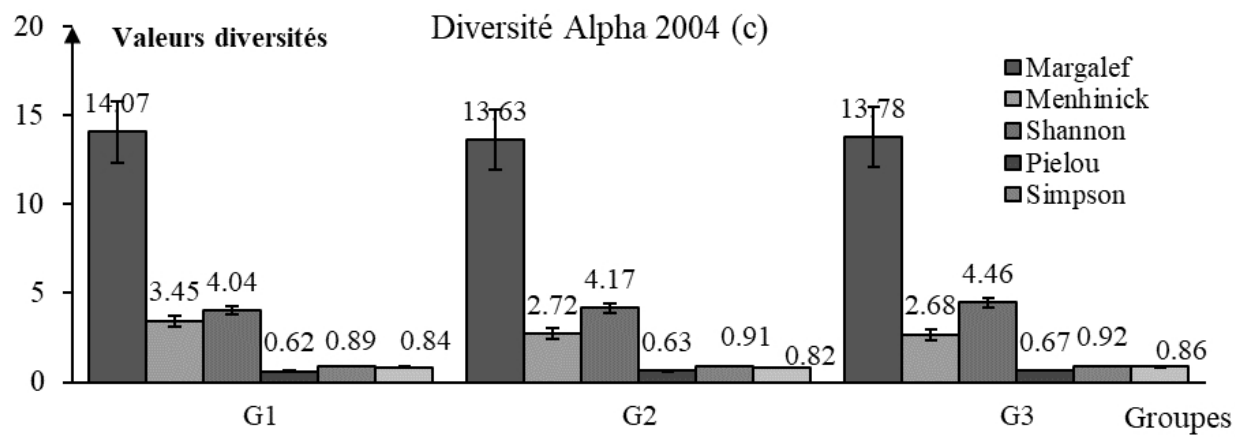




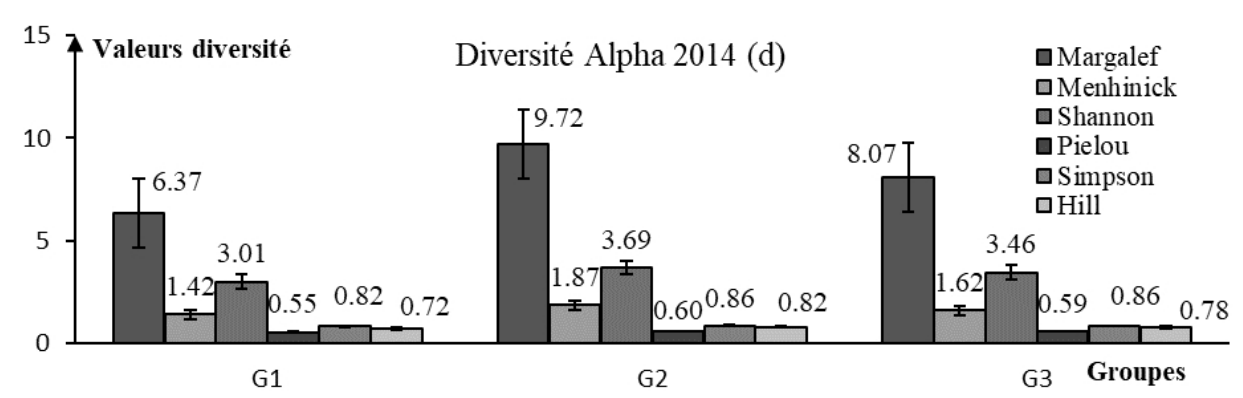

Figure 8 : Diversité Alpha de 2004 (k) et 2014 (1) des sites de Guidan Tawayé- Korin Habdjia.

- En 2004, la diversité spécifique des groupes G2, G3 (99, 101 espèces) est plus élevée que celle de G1 (94 espèces). Mais la tendance s'inverse au niveau des indices de Margalef avec respectivement 13,63 et 13,78 pour G2 et G3 et 14,07 pour G1. Cette tendance se poursuit au niveau de l'indice de Menhinick avec 2,72 et 2,68 pour G2 et G3 contre 3,45 pour G1 (Figure 8 : (c)). Il n'y a pas une différence significative au niveau de la diversité spécifique de différents groupes, mais l'abondance des espèces de $\mathbf{G 2}$ et G3 vaut presque le double de celle de G1. La diversité spécifique n'est pas proportionnelle à l'abondance en 2004.

Quant aux données de 2014, les diversités spécifiques les plus élevées sont rencontrées respectivement dans les groupes G2, G3 et G1 avec 72, 59 et 45 espèces. Cette tendance se maintient au niveau de la diversité de Margalef avec 9,$72 ; 8,07$ et 6,37 . Elle se poursuit aussi au niveau de l'indice de Menhinick avec respectivement 1,$87 ; 1,62$ et 1,42 (Figure 8: (d)). La diversité spécifique évolue dans le même sens que l'abondance dans l'ensemble de groupement de 2014. Elle est proportionnelle à l'abondance en 2014.

La diversité spécifique est plus élevée dans les groupes G2 et G3 que dans G1 en 2004 et 2014. Cette tendance se maintient au niveau de Margalef, et Menhinick 2014, mais s'inverse au niveau de ces mêmes indices en 2004.

- La diversité de Shannon-Weaver est élevée dans tous les groupements en $2004(4,22 \pm 0,217 \mathrm{bits})$ et relativement élevée en 2014 $(3,38 \pm 0,345)$, donc un nombre important d'espèces ou de fréquences peu différentes entre les espèces rencontrées dans la végétation (Figure 8 : (c) et (d)).

- L'équitabilité de Pielou au niveau de tous les groupements s'éloigne plus de zéro en $2004(0,638 \pm 0,02645)$, qu'en $2014(0,58 \pm 0,264)$ (Figure 8 op cit.), l'ensemble des relevés renferme des espèces dominantes (codominantes) ou très rares.

Les valeurs élevées de l'indice de Shannon et d'équitabilité de Pielou permettent de conclure une diversité spécifique relativement 
importante au niveau des ZSP, des champs et des zones mixtes de la vallée de Goulbi N'Kaba. Ces zones n'ont pas subi beaucoup de perturbations ces dix (10) dernières années. Les pratiques agricoles consistent à épargner des espèces telles que Faidherbia albida (Del.) A. Chev., Hyphaene thebaica (L.) Mart., Alysicarpus ovalifolius (Schum. Et Thonn.) J. Léonard. dans les champs et Acacia tortilis (Forsk.) Hayne sub sp. raddiana (Savi.) Brenan. en plus est disséminé par zoochorie dans les ZSP.

- la probabilité que deux individus sélectionnés au hasard appartiennent à la même espèce est élevée dans tous les groupes $(\mathrm{G} 1, \mathrm{G} 2, \mathrm{G} 3)$ en 2004 et 2014 car leur indice de diversité de Simpson moyen est élevé avec respectivement $0,91 \pm 0,0152$ et $0,85 \pm 0,023$ (Figure 8 : (c) et (d)). Les espèces abondantes sont importantes dans tous les groupements.

- Il existe une forte diversité de Hill en 2004 et 2014, car la valeur moyenne des différents groupes est respectivement de 0,84 $\pm 0,02$ et $0,77 \pm 0,050$ (Figure 8 op cit.). Il y a un grand nombre d'espèces abondantes, mais aussi d'espèces rares dans l'ensemble des groupements.

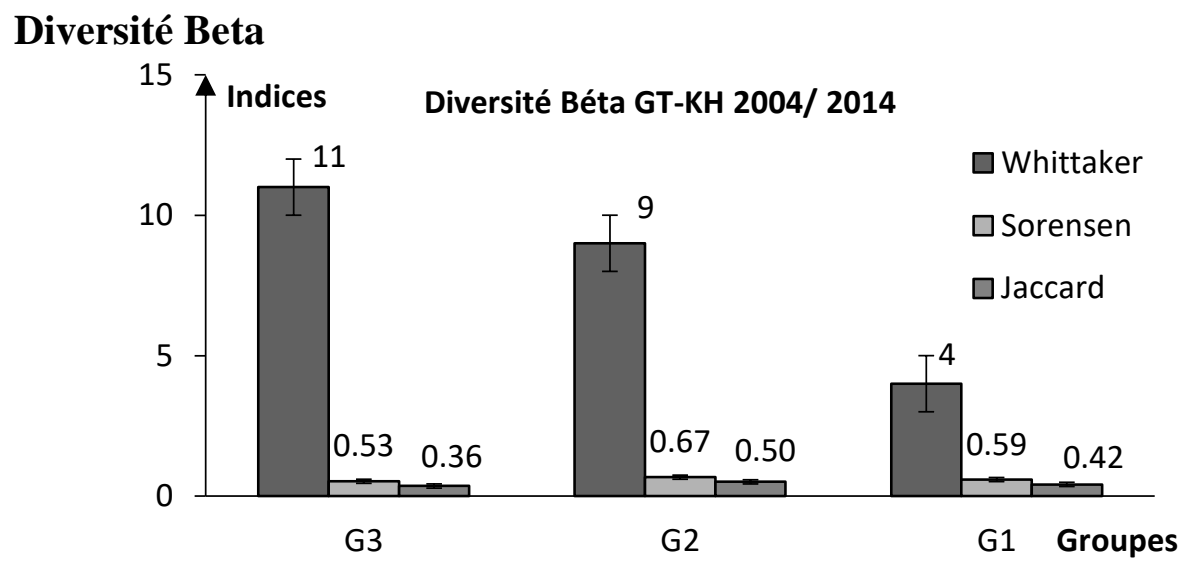

Figure 9 : Diversité Beta interdécennales (2004- 2014) des sites de Guidan Tawayé- Korin Habdjia.

- L'indice de Whittaker: il n'y a pas une forte diversité entre l'ensemble des groupements (G1, G2, G3) de 2004 et ceux de 2014, car leur indice de Whittaker moyen est de $8 \pm 4$ (Figure 9). Il y a une similarité entre les différents groupements de 2004 et ceux de 2014.

- L'indice de Jaccard, J : est relativement élevé, car sa valeur moyenne est de $0,425 \pm 0,0748$, donc un nombre important d'espèces se rencontre dans les deux groupements (2004- 2014) évoquant ainsi leurs biodiversités faibles (Figure 9).

- L'indice de Sørensen, indique qu'il y a une similarité entre les groupements (G1, G2 et G3) de 2004 et ceux de 2014 (Figure 9), car leur indice de Sørensen moyen 0,594士0,07315 est supérieur à 0,5. 
Il n'y a pas une forte diversité entre les groupements G1, G2, G3 de 2004 et ceux de 2014, car ils partagent plusieurs espèces communes. Il n'y a pas eu de perturbation significative au sein de la diversité interdécennale (2004-2014) comme le montre les indices de Whittaker, Jaccard et Sørensen.

\section{Discussion}

Familles, genres : La diversité spécifique de 2004 (150 espèces) est supérieure à celle de 2014 (99 espèces). Mais il y a une similarité au niveau de la proportion des ligneux et d'herbacées avec leur $\mathrm{p}$-value respective de 0,721 et 1 . Le nombre de famille de 2004 (40) est supérieur à celui de famille 2014 (30). Il y a une différence significative au niveau de la proportion de Poaceae et de Fabaceae avec p-value respectif de 0,0114 et 0,049. La même tendance se poursuit au niveau de genres (avec 105 en 2004 et 74 en 2014).

MNS et groupements : L'ensemble de relevés gardent pratiquement leur position par rapport aux axes sauf quelques-uns. Ceci est dû au nombre d'espèces rares qu'ils en possèdent. D'autres relevés s'écartent de leur groupe. Ceci est dû au nombre d'espèces plus élevé, qu'ils en possèdent par rapport aux autres du même groupe.

Le groupe G1 à Calotropis procera (Ait.) R. Br. et Jacquemontia tamnifolia (L.) Griseb. en 2004 est remplacé par celui à Acacia tortilis (Forsk.) Hayne subsp. raddiana (Savi.) Brenan. et Aristida mutabilis Trin. avec un taux élevé de Sida cordifolia L. en 2014. Ceci est dû au surpâturage de la zone qui favorise la dissémination (Forget, 1988) par desmochorie de diaspore de Aristida mutabilis Trin. et de l'inappétence de Sida cordifolia L.à l'état vert par les ruminants (Djibo et al., 2016).

Le groupe G2 à Acacia nilotica (L.) Willd. ex Del. subsp. nilotica et Peristrophe bicalyculata (Retz.) Nees., est remplacé par le groupe G2 à Calotropis procera (Ait.) R. Br. et Eragrostis tremula Steud. Ceci est dû au fait que les pluviométries de ces dernières décennies sont déficitaires. En plus les pluies précoces favorisent le développement des graminées, alors que les pluies tardives favorisent celui des légumineuses (Diallo, 1975 ; Boudet, 1975). Le groupe G3 à Leptadenia pyrotechnica (Forssk.) Decne. et Schoenefeldia gracilis Kunth. est remplacé par celui à Balanites aegyptiaca (L.) Del. et Glinus oppositifolius (L.) A. DC. avec une proportion élevée d'Alysicarpus ovalifolius (Schum. et Thonn.) J. Léonard. Ceci est dû à l'ensemencement d'Alysicarpus ovalifolius (Schum. et Thonn.) J. Léonard suivi de sa mise en défens de certains sites de la ZSP en 2003 (Saadou et al., 2004). La présence de Glinus oppositifolius (L.) A. DC. est due au fait que ces diaspores ont été apportées la vallée de Goulbi N'Kaba dans sa partie active. 
L'abondance évolue avec la diversité spécifique, les indices de Margalef et Menhinick en 2014, mais non en 2004.

Au niveau de la diversité Alpha, l'indice de Shannon-Weaver est élevé (Tonon, 2014) dans tous les groupes (G1, G2, G3) en 2004 et en 2014. Il y a régularité du recouvrement entre les espèces. Ces zones ont subi peu de perturbations cette dernière décennie (Soumon et al., 2007). Les conditions sont moyennement favorables pour l'installation d'autres espèces (Toko, 2008).

L'indice de diversité de Simpson, est élevé, il y a un nombre important d'espèces abondantes. Le fait d'ajouter des espèces rares à un échantillon, ne modifie pratiquement pas la valeur de l'indice de diversité (Grall et Hily, 2003).

L'indice de diversité de Hill est élevé dans l'ensemble de groupements (G1, G2, G3) de 2004 et 2014. Celui-ci est le plus pertinent dans la mesure où intègre les deux autres indices (Shannon et Simpson) (Grall et Hily, 2003). Il permet d'avoir une vue plus précise de la diversité observée. Donc la diversité est relativement élevée au niveau de la vallée de Goulbi N'Kaba. L'utilisation des trois indices conjointement a permis d'en extraire un maximum d'informations et de mieux comprendre la structure des communautés (Grall et Hily, 2003). Les indices de Shannon-Weaver, Simpson et Hill ont permis de mesurer les effets des traitements par l'ensemencement (Thomas et al. 1999), les pratiques agroforestières et pastorales sur la diversité floristique.

Au niveau de la diversité Beta, l'indice de Whittaker montre une forte diversité en 2004 et 2014, et une similarité entre les groupements de 2004 et ceux de 2014. Il compare les diversités des espèces entre les écosystèmes et les communautés. Il donne une mesure qualitative de la biodiversité et permet de voir son évolution à travers les changements des facteurs environnementaux (Soudant et Belin, 2011).

L'indice inter décennal de Jaccard pour l'ensemble de groupements indique une biodiversité faible, les conditions environnementales et les compositions floristiques sont similaires (De Bello, 2007 ; Chevrier et Machon, 2014).

Les groupements G1, G2, G3 de 2004 et ceux de 2014 partagent plusieurs espèces communes, comme en démontre l'indice de Sørensen. La pression sur la ZSP est faible et que les agriculteurs pratiquent un système agroforestiers qui leur permet d'épargner des espèces ligneuses telles que Hyphaene thebaica (L.) Mart., Faidherbia albida (Del.) A. Chev. (Diop et al., 2005), et que dans les ZSP l'espèce Acacia tortilis (Forsk.) Hayne subsp. raddiana (Savi.) Brenan. est fortement disséminée par les animaux (Masharabu et al., 2010). 


\section{Conclusion}

Cette étude a permis de rendre compte du degré de l'évolution interdécennale de la végétation de la vallée de Goulbi N'Kaba. Elle a concerné l'évolution de la diversité spécifique interdécennale, des ligneux, des herbacées, de familles et des genres. Les familles et les genres de 2004 (40 ; 105), sont légèrement plus élevés que ceux de 2014 (30 ; 74). Elle a été faite à travers la détermination de différents indices de diversité, Alpha et Beta. La diversité spécifique est plus élevée dans les formations steppiques des champs (G2) et les formations des ZSP (G3) que dans les formations steppiques mixtes (G1) en 2004 et 2014. Cette tendance se maintient au niveau de Margalef, et Menhinick en 2014, mais s'inverse au niveau de ces mêmes indices en 2004. C'est ainsi, qu'il a été constaté que la biodiversité végétale de la vallée de Goulbi N'Kaba est importante en 2004 et relativement importante en 2014. Qu'elle soit dans la ZSP, les zones mixtes ou dans les champs. Mais la diversité de 2014 a légèrement régressé par rapport à celle de 2004.

Quant à la diversité beta, elle est faible, due à la présence de plusieurs espèces communes en 2004 et 2014. Il n'y a pas une forte diversité entre les groupements des formations steppiques mixtes, des champs steppiques et des ZSP steppiques de 2004 et ceux de 2014, car ils partagent plusieurs espèces communes. Les indices de Whittaker, Sørensen et Jaccard en ont démontré. Il n'y a pas eu de perturbation significative au sein de la diversité interdécennale (2004-2014) de la vallée de Goulbi N'Kaba, comme le montre les indices de Whittaker, Jaccard et Sørensen.

\section{References:}

1. Ahmed, O., \& Rouscoua, B. (2001). Bilan et analyse des aspects hydro - environnementaux, écologiques et socio-économiques du bassin du fleuve Niger au Niger. Analyse diagnostique du bassin du fleuve Niger. MRE/ FEM/PNUD. Niger.

2. Boudet, G. (1975). Manuel sur les pâturages tropicaux et les cultures fourragères ( $2^{\text {ième }}$ édition). Ministère de la coopération. Institut d'Élevage et de Médecine Vétérinaire des Pays Tropicaux. Manuels et Précis d'Élevage.

3. Braun-Blanquet, J. (1932). Plant sociology. Macgran-Hill, New-York et London, 330p.

4. Chamard, P. C., et Courel, M. F. (1999). La forêt sahélienne menacée. Sécheresse, $\mathrm{n}^{\circ} 1$; vol 10 : (pp. 11-25).

5. Chapman, A. C. (1995). Primate seed dispersal. Coevolution and conservation implications. In: Bawa K.S. \& Hadley M., eds. Reproductive ecology of tropical forests plants. Paris: UNESCO, (pp. 30-50). 
6. Chevrier, M., \& Machon, N. (2014). Evaluation des récents changements de la diversité floristique en France. Muséum National d'Histoire Naturelle. ISUP.

7. Clarke, K. R., \& Ainsworth, M. (1993). A method of linking multivariate community structure to environmental variables. Marine Ecology Progress Series. 92: (pp. 205-219).

8. Clarke, K. R. (1993). Non-parametric multivariate analyses of changes in community structure. Australian Journal of Ecology. 18: (pp. 11714)

9. D’Avignon, H., Périé, C., Gerardin, V., \& Ouemet, R. (2000). Utilisation des indices de Pielou pour caractériser la diversité des types de végétation dans une aire commune de la forêt boréale. Note de recherche numéro 107. Ministère des Ressources Naturelles. Québec. ISBN 2-550-36681-6. Québec.

10. Daget, P., \& Poissonet, J. (1971). Une méthode d'analyse phytologique des prairies. Ann. Agron., 22, 1, (pp. 5-41).

11. Daget, P., \& Poissonet, J. (1991). Prairies et pâturages, méthodes d'études. Montpellier, France, Institut de Botanique.

12. De Bello, F. \& al., (2007). Grazing effects on the species-area relationship : Variation along a climatic gradient in NE Spain.-Journal of Vegetation Science 18 (2007) : (pp. 25-34).

13. Diallo, A. K. (1975). Considération sur les études de cartographie des pâturages naturels en République du Sénégal. In Inventaire et cartographie des pâturages tropicaux africains. Communication CIPEA. (pp. 79-81).

14. Diop, M., Kaya, B., Niang, A. \& Olivier, A. (2005). La biodiversité des espèces ligneuses : diversité arborée et unités de gestion du terroir dans le Cercle de Ségou, au Mali. ICRAF Working Paper $\mathrm{n}^{\circ} .10$. Nairobi : World Agroforestry Centre.

15. Djibo, I., Mamman, M., Bakhoum, A., Sarr, O., Marichatou, H., \& Akpo, L. E. (2016). Évaluation de l'importance du parcours Gadoudhé, dans l'alimentation du bétail de la commune rurale de Fabidji au Niger. ISSN 1997-5902. Journal of Applied Biosciences 106 : (pp. 1026610273).

16. Dufrêne, M., \& Legendre, P. 1997. Species assemblages and indicators species : the need for a flexible asymmetrical approach. Ecological Monographs, n67, p 345-366.

17. Forget, P. M. (1988). Dissémination et régénération naturelle de huit espèces d'arbres en forêt guyanaise. Biologie Végétale Tropicale. Thèse de Doctorat de l'Université Paris 6. 245p.

18. Gounot, M. (1969).- Méthodes d'études quantitatives de la végétation. (ed.), Masson, Paris. 
19. Grall, J., \& Hily, C. 2003. Traitement des données stationnelles (faune). FT-10-2003-01.doc.

20. Grall, J. \& Coïc, N. (2005). Synthèse des méthodes d'évaluation de la qualité du benthos en milieu côtier. Ifremer. DYNECO/ VIGIES/ 0613/ REBENT.

21. Hill, M. O. (1997). An eveness statistic based on abundance-weigthed variances of species proportions. Oikos 79, (pp. 413-416).

22. Ichaou, A. (2005). Capitalisation des outils techniques et méthodologiques développés par le PAFN pour la mise en gestion des principales formations forestières nigériennes. Projet d'Aménagement des Forêts Naturelles (PAFN). CIRAD. Louis Berger. Ministère de l'Hydraulique, de l'Environnement et de la lutte contre la désertification. Direction de l'environnement. République du Niger.

23. INS. (2014). Répertoire National des Localités (ReNaLoc), $4^{\text {ième }}$ RGP/H, 2012.Ministère de Finance, République du Niger.

24. Jouve, Ph. (1991). Usages et fonctions de la jachère en Afrique de l'Ouest et au Maghreb. In : Floret, C., Serpantié G. éds, La jachère en Afrique de l'Ouest. Paris, France, Orstom, (pp. 55-66).

25. Kilian J., \& Feau C. (1976). Opération Gerdat-Maradi (Niger). Reconnaissance morphopédologique de la zone de Mayahi. Compte rendu de la mission d'appui. Gerdat-IRAT.

26. Le Houérou, H. N., (1997). Climate, flora and fauna changes in the Sahara over the past 500 million years. Journal of Arid Environments. 327, rue de Jussieu, F 34090, Montpelier, France. 37: (pp. 619-647).

27. Legendre, P., \& Legendre, H. (1998). Numerical ecology: Developments Environmental Modelling. Volume 20. Elsevier : (pp. 235-245).

28. Magurran, A. E. (2004). Measuring Biological Diversity. Blackwell Science Inc. LTD. ISBN0-632-05633-9. Blackwell publishing. 063205633-9. 256 pages.

29. Mahamane, A. (2005). Etude floristique, phytosociologique et phytogéographique de la végétation du Parc Régional du W du Niger. Acta Botanica Gallica, $153: 2, \quad 265-269$, DOI : 10.1080/ 12538078.2006.10515543.

30. Masharabu, T., Noret N., Lejoly J., Bigendako M. J., \& Bogaert J. (2010). Étude comparative des paramètres floristiques du Parc National de la Ruvubu, Burundi. Géo-Éco-Trop., 34 : (pp. 29-44).

31. MC Cune B., Grace J. B., and Urban D. L. (2002). Analysis of Ecological Communities. MjM Software Design. Gleneden Beach. Oregon. 97388 USA. 300p. 
32. MDD (Ministère du Développement Durable). (2015). La Convention sur la diversité biologique» [archive], sur www.developpementdurable.gouv.fr (consulté le 28 mai 2015).

33. Peltier R., Duhem, S. C., \& Ichaou, A. (2008). Valoriser les produits du palmier doum pour gérer durablement le système agroforetier d'une vallée sahélienne du Niger et éviter sa désertification. Vertigo. Volume 8, Numéro 1.

34. Pielou, E. C. (1969). An introduction to mathematical ecology. New York: Wiley Inter Science.

35. Puig, H. (2001). Diversité spécifique et déforestation : l'exemple des forêts tropicales humides du Mexique. Revue éditée par le Cirad-forêt ; Bois et forêts des tropiques, $\mathrm{N}^{\circ} 268$ (2) $2^{\text {ième }}$ trimestre, (pp. 41-57).

36. R Development Core Team (2008). A language and environment for statistical computing. R Foundation for Statistical Computing, Vienna, Austria. ISBN 3-900051-07-0, URL http://www.R-project.org.

37. Saadou, M., \& Garba, M. (1997). Etude sur l'intégration des objectifs nutritionnels de la foresterie dans la sécurité alimentaire au Niger. Rapport provisoire.

38. Saadou, M. (1998). Evaluation de la biodiversité biologique au Niger : Eléments constitutifs de la biodiversité végétale. Conseil National de l'Environnement pour un Développement Durable SE/ CNEDD. Projet NER/ 97 / G 31 / A / 1 G / 99 "Stratégie Nationale et plan d'action Diversité Biologique". Niger.

39. Saadou, M. (2004). Etat des lieux de la diversité végétale et mise en place du dispositif de suivi environnemental du PAFN au niveau des massifs prioritaires de Baban Rafi (Madarounfa), du Goulbi N'kaba (Mayahi), Marigouna Bella (Dosso) et Onsolo (Téra); Projet Aménagement des Forêts Naturelles (PAFN). Niger.

40. Sarr O., Ngom D., Bakhoum A., \& Akpo L. E. (2013). Dynamique du peuplement ligneux dans un parcours agrosylvopastoral du Sénégal. Vertigo, Volume 13, Numéro 2.

41. Seydou, A., \& Saley, G. (1989). Pré-identification des douméraies du Goulbi N'Kaba dans le département de Maradi. Rapport Direction de l'Environnement. $46 \mathrm{p}$.

42. Shannon, C. E., \& Weaver, W. (1949). The mathematical theory of communication. Urbana, IL. University of Illinois Press.

43. Sonke, B. (1998). Études floristiques et structurales des forêts de la réserve de faune du Dja (Cameroun). Thèse de doctorat : Université Libre de Bruxelles (Belgique).

44. Sørensen, T. (1948). A method of establishing groups of equal amplitude in plant sociology based on similarity species content and its application to analyses of the vegetation on Danish commons. 
Biologist Skrifter Kongelige Danske Videnskabernes Selskab. 5 : (pp. 1-34).

45. Soudant, D., \& Belin, C. (2011). Note sur l'approche statistique de la diversité en écologie. Application à l'indice de composition pour le phytoplancton. Ifremer. Centre de Nantes. Dyneco/ Vigies/ 11-02/ DS. 46. Sounon, B., Sinsin, B., \& Goura, Soulé, B., (2007). Effets de la dynamique d'occupation du sol sur la structure et la diversité floristique des forêts claires et savanes au Bénin. Tropicultura. 25, 4, (pp. 221-227).

47. Thomas, S. C., Halpern, C. B., Falk, D. A., Liguori, D. A., \& Austin, A. K., (1999). Plant diversity in managed forests: understory responses to thinning and fertilization. Ecological Application 9 : (pp. 864-879).

48. Toko, I. I. (2008). Etude de la variabilité de la biomasse herbacée, de la phénologie et de la structure de la végétation le long des toposéquences du bassin supérieur du fleuve Ouémé au Bénin. Gestion de l'Environnement ; Dynamique des Ecosystèmes et Aménagement du Territoire. FLASH, UA-C, FST. Bénin.

49. Tonon A. (2014). Implantation et suivi scientifique d'un pilote récif artificiel dans la Réserve Naturelle de Saint-Martin. Université la Rochelle. 